Florida International University

FIU Digital Commons

8-9-2002

\title{
Design of a fast and resource-efficient fault management system in optical networks to suit real-time multimedia applications
}

Tirthankar Ghosh

Florida International University

Follow this and additional works at: https://digitalcommons.fiu.edu/etd

Part of the Computer Engineering Commons

\section{Recommended Citation}

Ghosh, Tirthankar, "Design of a fast and resource-efficient fault management system in optical networks to suit real-time multimedia applications" (2002). FIU Electronic Theses and Dissertations. 3931.

https://digitalcommons.fiu.edu/etd/3931

This work is brought to you for free and open access by the University Graduate School at FIU Digital Commons. It has been accepted for inclusion in FIU Electronic Theses and Dissertations by an authorized administrator of FIU Digital Commons. For more information, please contact dcc@fiu.edu. 
FLORIDA INTERNATIONAL UNIVERSITY

Miami, Florida

DESIGN OF A FAST AND RESOURCE-EFFICIENT FAULT MANAGEMENT SYSTEM IN OPTICAL NETWORKS TO SUIT REAL-TIME MULTIMEDIA APPLICATIONS

A thesis submitted in partial fulfillment of the requirements for the degree of

MASTER OF SCIENCE

in

COMPUTER ENGINEERING

by

Tirthankar Ghosh

2002 
To: Dean Vish Prasad

College of Engineering

This thesis, written by Tirthankar Ghosh, and entitled Design of a Fast and Resourceefficient Fault Management System in Optical Networks to Suit Real-time Multimedia Applications, having been approved in respect to style and intellectual content, is referred to you for judgment.

We have read this thesis and recommend that it be approved.

Kia Makki

Subbarao Wunnava

Jian Wang

Niki Pissinou, Major Professor

Date of Defense: August 9, 2002

The thesis of Tirthankar Ghosh is approved.

Dean Vish Prasad College of Engineering

Dean Douglas Wartzok University Graduate School

Florida International University, 2002 


\section{ACKNOWLEDGMENTS}

I hereby acknowledge that I am grateful to my advisor Dr. Niki Pissinou to help me successfully finish my thesis. This work would not have been possible without her able guidance and support. I also acknowledge the guidance of Dr. Kia Makki, Dr. Subbarao Wunnava, Dr. Gang Feng and Dr. Jian Wang who helped me in every step in completing my thesis.

I am also highly indebted to the Telecommunication and Information Technology Institute at FIU and its directors, Dr. Niki Pissinou and Dr. Kia Makki for giving me financial support in the form of Graduate Assistantship. I sincerely extend my warm regards to all my fellow colleagues without whose co-operation it would have been difficult for me to carry on with this research. 


\section{ABSTRACT OF THE THESIS \\ DESIGN OF A FAST AND RESOURCE-EFFICIENT FAULT MANAGEMENT \\ SYSTEM IN OPTICAL NETWORKS TO SUIT REAL-TIME MULTIMEDIA}

APPLICATIONS

by

Tirthankar Ghosh

Florida International University, 2002

Miami, Florida

Professor Niki Pissinou, Major Professor

Today's telecommunications networks are relying more and more on optical fibers as their physical medium. Currently the Wavelength Division Multiplexing technology enables hundreds of wavelengths to be multiplexed on a single fiber. Using this technology capacity can be dramatically increased, even to the order of Terabits per second. While WDM technology has given a satisfactory answer to the ever-increasing demand for capacity, there is still a problem which needs to be handled efficiently: survivability.

Our proposed fault restoration system optimized between restoration cost and speed. We extended the concept of Forward Equivalence Class (FEC) in Multi Protocol Label switching (MPLS) to our proposed fault restoration system. Speed was found to be in the order of 1 to 3 microseconds using predesigned protection, depending on the configuration of the system. Optimization was done between restoration speed and cost by introducing a priority field in the packet header. 
CHAPTER

PAGE

1.INTRODUCTION -

1.1 Background ---

1.2 Objective of thesis and problem statement -.-.-

1.3 Overview of approach -- 8

1.4 Target of application -

1.5 Scope of thesis -

1.6 Organization of thesis -

2. RELATED WORK - 10

3. DESIGN OF ALGORITHMS -..-16

3.1 Optical layer - its insurgence -- 16

3.2 MPLS extended to optical networks--19

3.3 Design--

3.3.1 Concepts and assumptions--

3.3.2 Algorithms - - 28

4. SIMULATION AND RESULTS -

4.1 Simulation Scenario - - 34

4.2 Results --- 36

4.3 Conclusion and Future Work -..- 38

BIBLIOGRAPHY - 


\section{LIST OF FIGURES}

FIGURE

PAGE

1.1 Line Protection Switched Unidirectional Self healing Ring ---_-_-_-_-

1.2 Path Protection Switched Unidirectional Self healing Ring ---------------4

1.3 Two Fiber Unidirectional Self healing Ring ---

1.4 Four Fiber Unidirectional Self healing Ring -

1.5 Dedicated Link Protection--

1.6 Shared Link Protection-- 6

1.7 Dedicated Path Protection - 7

1.8 Shared Path Protection --

3.1 WDM Mesh Network used in the design- 29

4.1 WDM Mesh Network -...-34 


\section{LIST OF ABBREVIATIONS}

WDM : Wavelength Division Multiplexing

OXC : Optical Cross Connect

WSXC : Wavelength Selective Cross Connect

WIXC : Wavelength Interchange Cross Connect

APS : Automatic Protection Switching

SHR : Self Healing Ring

SONET : Synchronous Optical Network

SDH : Synchronous Digital Hierarchy

USHR : Unidirectional Self Healing Ring

BSHR : Bidirectional Self Healing Ring

USHR/L : Link Protection Switched Unidirectional Self Healing Ring

USHR/P : Path Protection Switched Unidirectional Self Healing Ring

BSHR/2 : 2- Fiber Bidirectional Self Healing Ring

BSHR/4 : 4- Fiber Bidirectional Self Healing Ring

MPLS : Multi Protocol Label Switching

FEC : Forward Equivalence Class

ATM : Asynchronous Transfer Mode

IP : Internet Protocol

TCP : Transmission Control Protocol

OTN : Optical Transport Network

ITU : International Telecommunications Union

OCh : Optical Channel 
OMS : Optical Multiplexed Section

OAS : Optical Amplifier Section

IETF : Internet Engineering Task Force

LSP : Label Switched Path

LSR : Label Switched Router

OSPF : Open Shortest Path First

LDP : Label Distribution Protocol

RSVP : Resource Reservation Protocol

GMPLS : Generalized Multi Protocol Label Switching

RWA : Routing and Wavelength Assignment 


\section{Chapter 1 Introduction}

Today's telecommunications networks are relying more and more on optical fibers as the physical medium. The reasons are obvious. Optical networks can provide the right capacity that today's service providers are looking for, combined with optimum cost, high reliability and provision for scalability. As data over Internet are growing day by day, the service providers as well as the end-users are looking for fast and faulttolerant service, particularly to suit multimedia applications. Perhaps the most satisfactory solution lies in using optical fibers as the communication medium.

To utilize the capacity of a fiber more efficiently with a view to provide a solution to more and more demand for higher capacity, a technology known as Wavelength Division Multiplexing (WDM) emerges $[1,4,6,9,12]$. This technology enables several sources to transmit lightpath signals over a single fiber at different wavelengths (frequencies). In other words, several lightpaths at different wavelengths are multiplexed on a single fiber. Using this technology, capacity can be dramatically increased even to the order of Terabits per second $[1,8]$. Scientists from Bell Laboratories have carried out successful demonstrations of multiplexing hundred wavelengths over a single fiber, each wavelength modulated at $10 \mathrm{~Gb} / \mathrm{sec}$, thus providing a throughput of $1 \mathrm{~Tb} / \mathrm{sec}[1]$.

In WDM architecture several Optical Cross-Connects (OXCs) are connected by optical fibers. There are two types of OXCs available: Wavelength Selective Cross Connect (WSXC) and Wavelength Interchange Cross Connect (WIXC) [5]. In WSXC lightpath from an incoming fiber is routed to an outgoing fiber at the same wavelength. The node does not provide any wavelength conversion facility. This gives rise to a well- 
known problem called Wavelength Continuity Constraint. Discussion of this problem is outside the scope of this thesis. In contrast, the second type of Optical Cross Connects, i.e., WIXCs, solves the wavelength continuity constraint. WIXCs provide wavelength conversion facilities at the nodes.

WDM technology has given a satisfactory answer to the ever-increasing demand for capacity. But the technology has got an inherent problem with it - survivability $[1,5$, $6,8,10]$. Since hundreds of wavelengths are multiplexed on a single fiber, and hundreds of fibers comprise a single link, failure of a single link brings down several lightpath connections at the same time. This requires a very efficient fault restoration system which, on one hand, provides a fast restoration method to suit real-time applications and, on the other hand, utilizes the network capacity efficiently.

\subsection{Background}

Survivability in optical networks can be classified broadly into two types: restoration and protection $[1,5,6,8,10]$. Restoration means that the connections are routed to an alternative path which is computed after failure occurs. When the source node detects a failure, it computes an alternative path and switches the failed connection to that path. Restoration method can utilize the available resources in a network very efficiently, but it suffers from one big disadvantage: it takes longer time to restore a failed connection. Protection typically refers to the pre-establishment of a backup path which is used to switch a connection when the primary path fails. This provides a very fast survivability mechanism. Protection can be of two types: link-based protection and pathbased protection. In link-based protection, a backup path is calculated for every link 
whereas in path-based protection a single backup path for the entire path from source to destination is set up. The two most common protection mechanisms available today are Automatic Protection Switching (APS) and Self-healing Ring (SHR) [1].

APS, which is typically used to handle link failures, can be of three types: $1+1$, 1:1 and $1: \mathrm{N}$ [1]. In $1+1$ protection mechanism, every link has a dedicated protection link. The source node transmits in both the links. Whenever the destination node detects a failure, it switches over to the protection link. In 1:1 protection mechanism also, each link has a protection link which lies idle in normal condition. When a failure occurs, both source and destination nodes switch to the protection link. In 1:N protection mechanism, $\mathrm{N}$ working links share a single protection link. This is called shared link protection.

SHR architecture is typically used by Synchronous Optical Networks (SONET) [1]. It uses a ring architecture. SHR can be either unidirectional (USHR) or bi-directional (BSHR). USHR can also be classified into two types: line protection switched (USHR/L) and path protection switched (USHR/P). USHR/L works in the same way as FDDI, as shown in Fig 1.1. Here the two nodes on both sides of the failed path are responsible for switching. In USHR/P, signals are sent simultaneously in both paths. When one path fails, the nodes decide which signal is good and switches to that, as shown in Fig 1.2. Bidirectional SHRs typically use two architectures: two-fibered BSHR and four-fibered BSHR. In BSHR/2, half the capacity in each ring is reserved for protection, while in $\mathrm{BSHR} / 4$, two rings are used as working rings and the remaining two are protection rings. Fig 1.3 and 1.4 show $B S H R / 2$ and BSHR/4 respectively. 


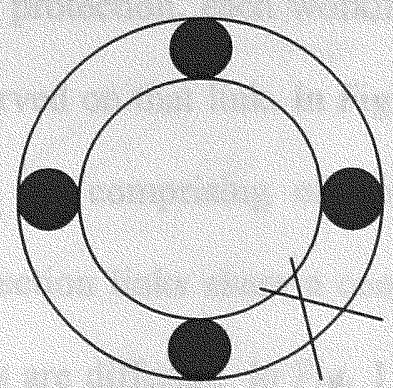

Fig. 1.1 Line Protection Switched Unidirectional Self healing Ring [1]

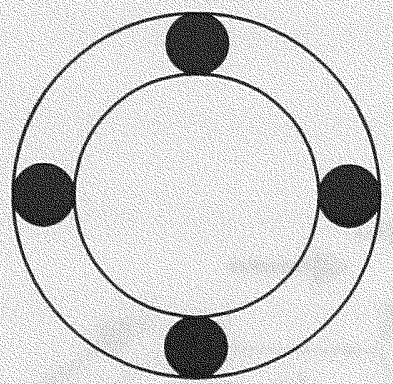

Fig. 1.3 Two Fiber

Bidirectional Self healing Ring [1]

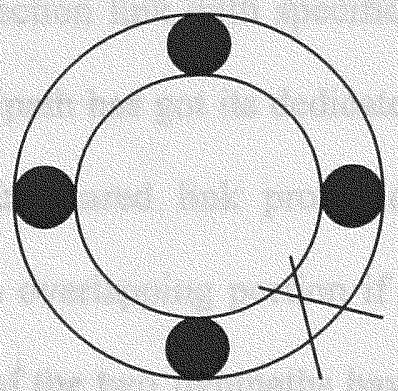

Fig. 1.2 Path Protection Switched Unidirectional Self healing Ring [1]

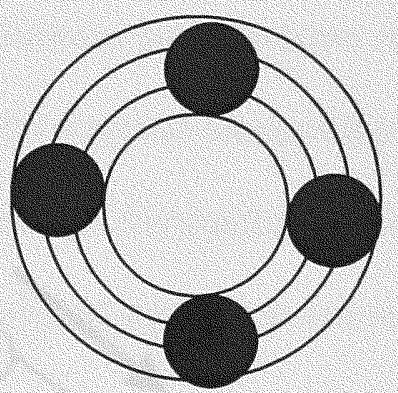

Fig. 1.4 Four Fiber

Bidirectional Self healing Ring [1]

Similar survivability schemes, as discussed earlier, can be used for WDM networks. The main concern here is that a single fiber carries hundreds of wavelengths. Hence a single fiber failure can result in the loss of hundreds of lightpath connections. Pre-designed protection mechanism in WDM networks can be broadly classified into link-based and path-based protection $[1,5,6,10]$. In link-based protection, each link has got its own protection link. This protection link can be dedicated or shared. In dedicated 
link protection, each working link has its own protection link with specific wavelength reserved on that link. In Fig 1.5, link 2-3 of the lightpath has got its dedicated protection path p1 comprising of links $2-7,7-8$ and $8-3$. In shared link protection, different protection links share a common wavelength on the overlapping portion if the working links are different. In Fig. 1.6, links 2-3 and 11-12 of the two lightpaths have protection paths p1 and p2 respectively which share a common link 7-8. The shared link 7-8 can share a common wavelength as the two working lightpaths are different.

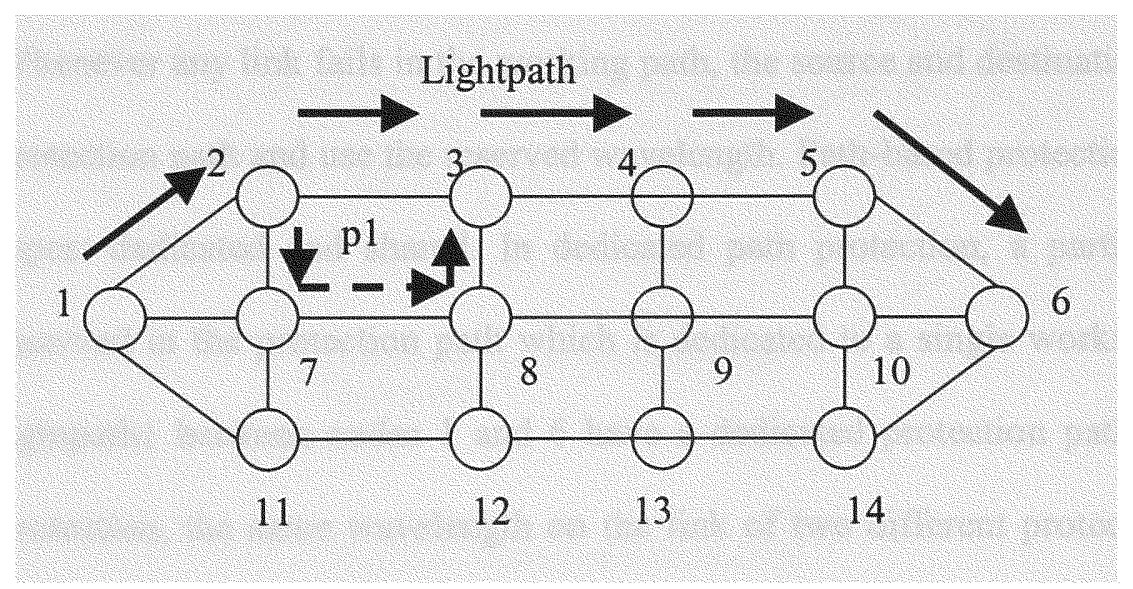

Fig. 1.5 Dedicated link protection 


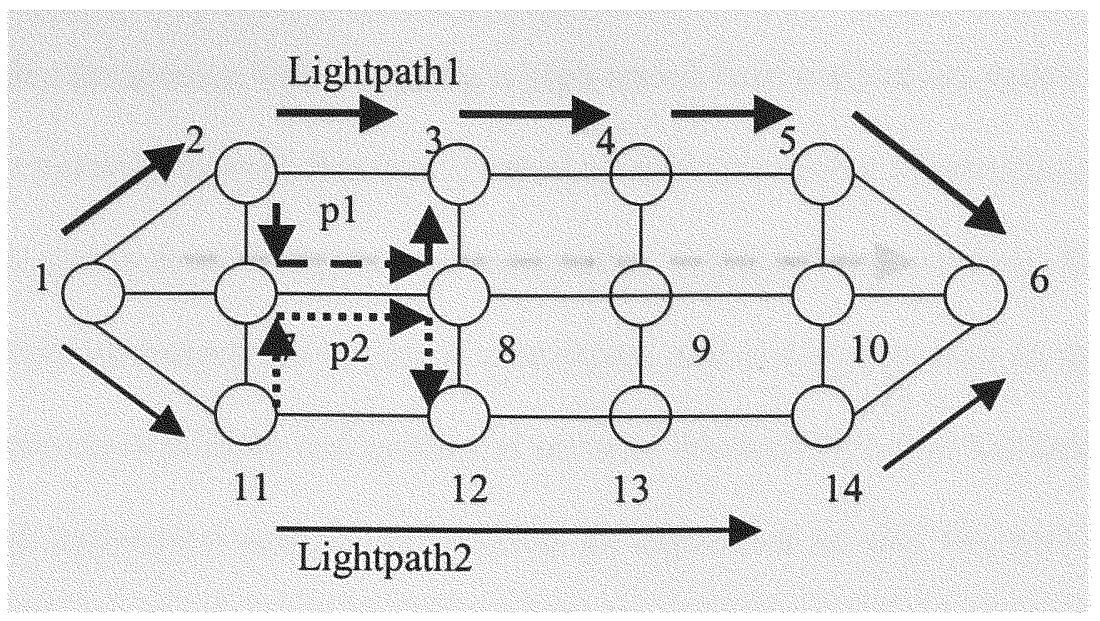

Fig. 1.6 Shared link protection

In path-based protection mechanism, each working path between a source and destination has its own protection path and wavelengths reserved along that path $[1,5,6]$. Whenever any link fails in the working path, the source and destination switch over to the protection path and use the reserved wavelength. Path-based protection can also be of two types: dedicated and shared. In dedicated path protection, a particular wavelength is reserved in the protection path which is dedicated to a single working path. In Fig. 1.7, lightpath1 between nodes 1 and 6 have a dedicated protection path p1. In shared path protection, the same wavelength on the link of two different protection paths is used if corresponding working paths are link-disjoint. In Fig. 1.8, working lightpaths 1 and 2 are link-disjoint, hence the protection paths $\mathrm{p} 1$ and $\mathrm{p} 2$ share the same wavelength along all the common links. 


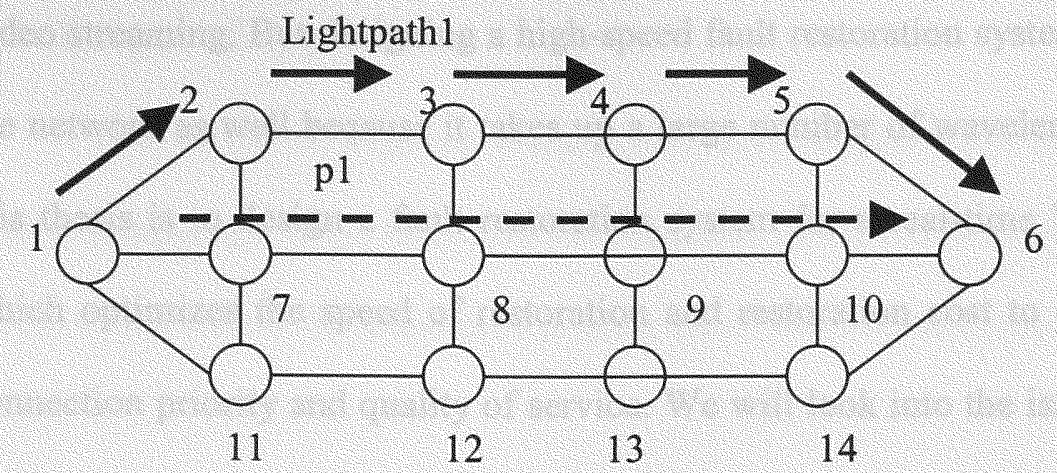

Fig. 1.7 Dedicated path protection

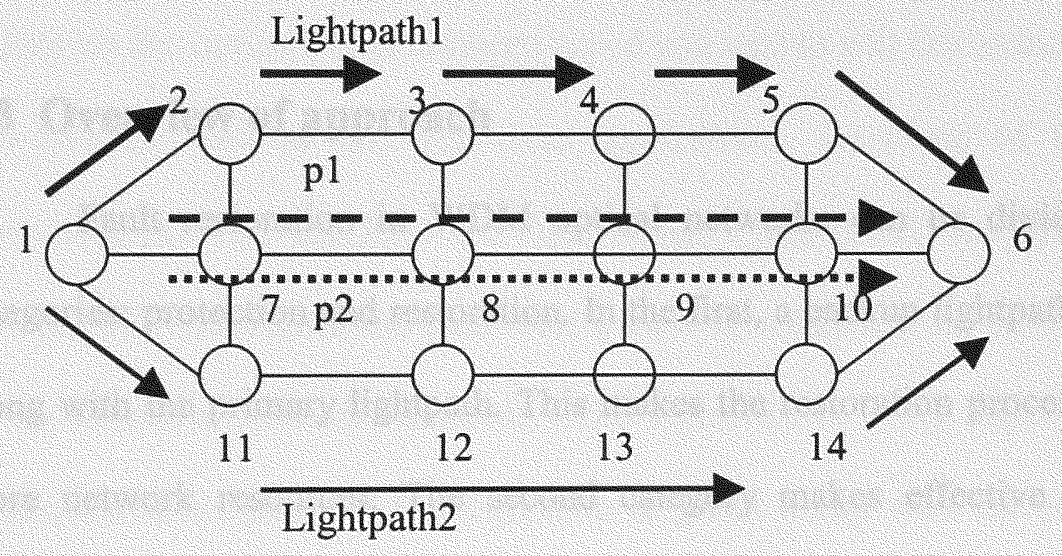

Fig. 1.8 Shared path protection

\subsection{Objective of the thesis and Problem Statement}

A fault-tolerant WDM network design has got several challenges. Most important of these are speed and cost. Speed is important in real-time multimedia applications like 
video streaming. But designing a high-speed fault restoration system increases the cost of the network as well because it takes up a large number of wavelengths. The objective of this thesis is to design a fault restoration system for a real-time WDM optical network which optimizes the speed of restoration and restoration cost to suit different levels of connection priority and quality of service. We will look into the issues of both protection and restoration and try to develop an algorithm to suit the real-time high-bandwidth application. However, we will not deal with any specific routing and wavelength assignment algorithm, and will assume that any such existing algorithm can be used in our fault restoration method.

\subsection{Overview of approach}

Fault restoration in WDM optical networks can be divided broadly into two categories: protection and restoration. In the first, a backup lightpath is set up beforehand along with the primary lightpath. This makes the restoration process faster, but takes up more network resources. The second category makes effective use of the available network resources, although a bit slower than the first one. This category finds out a restoration path after fault occurs and assigns wavelengths along that path. In this thesis, the fault restoration method is designed taking into consideration three categories of fault restoration: simultaneous transmission, wavelength reservation and normal restoration. The first two categories come under the protection scheme while the second one is the restoration. 


\subsection{Target of application}

The thesis is targeted towards the second-generation optical networks using WDM technology. Fault restoration is suggested at the WDM layer instead of the higher layers which makes the restoration process much faster to suit real-time applications.

\subsection{Scope of thesis}

Design of fast fault restoration system for WDM optical network is suggested in this thesis. The design is developed keeping in mind the challenges for optical network restoration like speed, cost, resource availability and reservation conflict. The focus is mainly on the optimization between speed and cost to suit the connection priority for both real-time as well as non real-time applications. Concepts of Multi Protocol Label Switching (MPLS) and Forward Equivalence Class (FEC) are extended to set up both service path and restoration path with a view to minimize the time of restoration as well as the available network resources. The algorithm is being implemented using $\mathrm{C}++. \mathrm{A}$ theoretical description of the algorithm is given along with the results from the actual implementation.

\subsection{Organization of the thesis}

The rest of the thesis is organized as follows. Chapter 2 covers related works in the field of optical network restoration. Chapters 3 deals with the design and the proposed algorithms. Finally, Chapter 4 discusses simulation and results and concludes the study. 


\section{Chapter 2 Related Work}

Survivability has been a hot research issue ever since the evolution of WDM technology. Survivability typically refers to maintaining the lightpath connection after a failure occurs. In traditional optical networks, before the evolution of WDM technology, failure of one fiber leads to the failure of only one lightpath connection. But in WDM optical networks, failure of one single fiber brings down hundreds of lightpath connections. Since the evolution of WDM technology, many researchers have tried to focus their research on this survivability issue.

Parallelly with the advent of WDM technology, another technology emerges to provide a fast switching mechanism in conventional IP networks. This is called Multi Protocol Label Switching (MPLS) [13]. With a view to provide a fast and efficient switching mechanism at the IP routers, this MPLS technology has been widely accepted in various research wings. Some work has also been done to extend the concept of MPLS to the survivability of WDM networks [12].

In [10], Didier Colle, et al. described the conventional IP-MPLS network survivability mechanism. Two methods have been discussed - restoration and protection (see chapter 1 for more details).

The same concepts of IP-MPLS survivability mechanism can be extended to WDM networks as well. Zhou and Subramanian in [1] have discussed several survivability mechanisms available for today's optical networks. This paper first discusses survivability in non-WDM networks which has been classified into predesigned protection and dynamic restoration. In predesigned protection, failed lightpath 
connections are switched to an alternate path which has been set up at the time of the primary path itself. The two most common protection mechanisms available today are Automatic Protection Switching (APS) and Self-healing Ring (SHR) (see chapter 1 for details).

In dynamic restoration survivability scheme, the protection path is calculated after the failure occurs. This scheme utilizes the available network resources quite efficiently, but takes longer time for fault restoration than pre-designed protection. Most of the researches have been concentrated on pre-designed protection and very little work has been done so far on dynamic restoration mechanism.

With the evolution of the WDM system, and the deployment of optical networks in the backbone, a new network layer has been introduced in the layered architecture. This layer, called the WDM layer, provides services to the upper layers like SONET, ATM and IP. With this multilayer architecture of WDM optical networks, an obvious question arises - in which layer to provide survivability? In [10], Didier Colle, et al. have discussed several single layer and multilayer survivability strategies. Single layer survivability strategies include survivability at the bottom layer and at the top layer. Recovery at the bottom layer has an inherent disadvantage in that any failure at the upper layers cannot be recovered. Similarly, recovery at the top layer requires the layer to have a macro view of all the functionalities at the lower layers. A feasible solution is to provide survivability at multiple layers. Didier Colle, et al. have discussed several such multilayer survivability schemes. One such approach is the uncoordinated approach. In this approach, restoration is carried out both at the top and bottom layers parallelly without any coordination between them. This is very simple to visualize as well as to 
implement. But it takes up spare resources at both the layers. Another multilayer survivability scheme is the sequential approach. In this approach, a layer hands over responsibility to the next layer when it finds out that it is unable to restore the fault. Several such approaches can be visualized - bottom-up approach, where the survivability starts at the bottom layer and moves up to the higher layers; and top-down approach, where the survivability starts at the top layer and moves down to the lower layers. How the responsibilities are handed over from one layer to another is beyond the scope of this discussion.

There is a third multilayer survivability strategy called integrated approach. In this approach the recovery scheme has got the full overview of the functionalities and resources in all the layers and accordingly decides when and in which layer to take action.

This multilayer survivability is also discussed by Fumagalli and Valcarenghi in [6]. They proposed a heuristic for a hybrid solution in which a portion of the traffic is protected by WSHR and the remaining portion by the IP layer restoration scheme. The problem they proposed to solve has been defined as the IP-aware wavelength minimum mileage problem. The total wavelength mileage is minimized jointly with an arbitrarily defined IP layer cost [6].

Finally, some case studies have been presented in [10]. The conclusions drawn from the case studies are - a) protection at the optical layer is more expensive than at the MPLS layer and the cost increases for local protection; b) cost is also more for dedicated protection than shared protection and the difference is more for local protection; c) although MPLS protection is cheaper than MPL(ambda)S protection and it also provides 
a fast protection method, MPLS protection has got a negative impact on the TCP output which depends upon the speed of switching; d) $1+1$ protection in the OTN layer leads to more expensive solution. In fact it is $1.7-1.8$ times more expensive than restoration, if no MPLS protection mechanisms are used. Path restoration is the cheapest solution. Moreover, when MPLS local protection is introduced, the overall network cost increases, especially for $1+1$ protection. Again path restoration is the cheapest solution. Hence, protecting against MPLS router failure with as much recovery as possible in the OTN layer, without proper precaution, may have a negative impact on the overall network cost.

Zhou and Subramaniam have discussed some advantages of WDM layer survivability in [1]. These include - speed, simplicity, effectiveness and transparency. Restoration at WDM layers is much faster than at the higher layers, it needs lesser coordination, uses the restoration capacity more efficiently and does not depend upon the protocols used in the higher layers.

In [3], Crochat, et. al. have discussed about the failure propagation problem which is defined as follows: a single resource failure at the WDM layer can cause simultaneous failure of several higher level layers. It may happen that the IP network is partitioned into several islands and restoration at the higher layers is impossible. They have developed a mapping algorithm to solve this problem. The solution will take into account the failure propagation problem at the higher layers due to a single resource failure at the WDM layer without changing the functionalities of the higher layer software.

In [8], Ramamurthy and Mukherjee have examined a problem for fault tolerant optical networks. The problem formulation is as follows: given a set of lightpaths and alternate routing table, to assign routes and wavelengths to each lightpath such that the 
lightpath is restored upon any single link failure without any blocking. They have considered both dedicated and shared path protection and formulated ILPs to solve the heuristics.

Similar problems are considered in [9] by Sahasrabuddhe et. al. They have considered both protection and restoration and have developed mathematical formulations for those fault management techniques. Their solution to the ILPs provides optimum virtual topology design and traffic flow assignment that maximizes the available network capacity. Comparison of the recovery time between protection and restoration has shown that shared path protection outperforms IP restoration in some cases like where there are single transmitter and receiver per wavelength per node.

Doverspike and Yates have discussed several issues for extending MPLS to optical network restoration. In [12] they have discussed several categories in optical network restoration and their relative pros and cons. In categoryl restoration, the path calculation, channel assignment and cross connect are done before failure occurs. In category2, path calculation and channel assignment are done before the failure but cross connect is carried out after failure occurs. In category3, only the path is calculated before, channel assignments and cross connects are done after connection failure. Lastly, in category4, all the steps are done after failure occurs in the network.

It is quite obvious to conclude that categoryl restoration is very fast, but on the other hand, it is very costly as it takes up a large number of network resources. In fact, Doverspike and Yates have claimed in their work that, in case of dedicated restoration, the restoration overbuild (ratio of required restoration channel to service channel) is 180 percent. They have also claimed that category 2 restoration is very difficult to implement. 
Category 4 is a fully dynamic restoration scheme and utilizes network resources very efficiently. It has got one disadvantage in that it is slower than the other schemes. Category 3 has been taken to be the most economical and practical method among all the schemes discussed.

Thus, it can be concluded that in a multilayered WDM optical network, the best layer to have the restoration functionalities is the WDM layer. The rest of the thesis will discuss restoration schemes at the WDM layer and will attempt to develop algorithms for fault restoration to optimize between speed and cost taking into consideration all the categories of restoration discussed earlier. 


\section{Chapter 3 Design of Algorithms}

\subsection{Optical layer - its insurgence}

Ever since the beginning of computer networks, all the network functionalities have been distributed in several layers. In the early 1980's, the International Standards Organization proposed a seven-layer architecture of the traditional networks. Nowadays, most networks use a five-layer TCP/IP model which distributes the network functionalities into five well-defined layers.

The first generation optical networks saw the evolution of the SONET/SDH layer in the classical layer hierarchy. As demand for bandwidth grew up more and more, copper cable was replaced by optical fiber as the transmission medium. Although optical fiber acted only as the physical carrier, most of the switching and processing of data are done by the electronic circuitry at each node. This type of network came to be known as first generation optical networks [14]. Soon, even the first generation optical networks became insufficient to cope up with the rising demand for bandwidth. To provide more capacity to the fibers, the well known technique of multiplexing was extended to optical networks, where several wavelengths were multiplexed on a single fiber. This technology came to be known as wavelength division multiplexing (WDM) $[1,3,5,6,10,14]$. Besides WDM technology, researchers came up with the idea of faster processing of data at each node than that provided by first generation networks. In first generation networks, the electronic circuitry at each node was meant to process data not only destined for that particular node, but also destined to all other nodes. It was thought of that if data destined for other nodes are passed instead of being processed, then the load on the electronic 
circuits at each node can be reduced by a considerable extent. This gave the idea of second generation optical networks [14]. Most of the first and second generation optical network functionalities fall mostly on the physical or data link layer [14]. A common example of first generation optical network is ATM over SONET. The ATM network treats the SONET network in its data link layer. The SONET network itself has its own physical, data link and network layers.

With the evolution of WDM, the International Telecommunications Union (ITU) defined a separate layer known as the optical layer. The functionalities of this optical layer are very similar to that of the SONET layer and are divided into three sub-layers Optical Channel (OCh) layer, Optical Multiplex Section (OMS) layer and the Optical Amplifier Section (OAS) layer [14]. The optical channel layer is concerned with the end to end routing of a lightpath. A lightpath consists of several links which in turn has several lightpaths multiplexed in it. The OMS layer is concerned about the point-to-point link in a route of a lightpath. The OAS layer takes care of the link segment between the optical amplifiers.

Since the evolution of the optical layer, researchers have proposed several schemes for network survivability in the optical layer as well as in the higher layers $[1,3$, $6,7,10]$. One important aspect in this design is assignment of various functionalities to the optical layer and the higher layers such that no resources are duplicated. An appropriate interface is to be designed to carry out efficient co-ordination between different layers. Several such approaches are discussed in [1]. The bottom-up approach involves recovery at the lowermost layer and gradually moves up to higher layers. Time to move to each higher layer is controlled by a timer. If the timer expires before the fault 
is restored by a lower layer, responsibilities are moved up to the higher layer. Exact reverse happens in top-down approach. Here recovery starts at the highest layer and moves down to the lower layers on the expiry of a timer. Another approach involves recovery at any intermediate layer and moving up or down according to the survivability design.

There are several advantages of carrying out recovery at the optical layer [1]. Foremost of these are speed and resource-efficiency. Recovery at the optical layer is much faster than that at the higher layers. The reason is quite simple. Upon occurrence of a failure, the nodes can act very fast without the interference of any higher layer. Recovery at the optical layer also utilizes the network resources very efficiently. In addition, optical layer recovery is much simpler as it needs much less co-ordination and is independent of any higher layer protocol.

Recovery at the optical layer can be broadly classified into predesigned protection and dynamic restoration $[1,5,6,8,10]$. In predesigned protection, a restoration path is set up and wavelength reserved along that path at the same time when the service path is set up. On the other hand, in dynamic restoration, the source node finds a restoration path and assigns wavelength to it after the occurrence of a failure. Predesigned protection is much faster than dynamic restoration, but takes up a lot of network resources. Predesigned protection can be of two types - path-based and link-based. In path-based protection, each service path has got a restoration path which can be either dedicated to that service path or shared between a number of paths. Similarly, in link-based protection, each working link has got either a separate protection link or a shared link. Details of 
dedicated and shared path-based and link-based protection were already discussed in details in chapter 1.

\subsection{MPLS extended to optical networks}

Multi Protocol Label Switching (MPLS) was introduced as an effort to provide fast switching to the conventional IP networks. In 1997 the Internet Engineering Task Force (IETF) formed the MPLS working group to develop a standardized switching protocol in line with Asynchronous Transfer Mode (ATM) for fast switching of IP packets. Today MPLS has been widely accepted as a research standard by all the industries.

Operation of MPLS is quite simple. MPLS enabled routers, called Label Switched Routers (LSRs), forward each packet on the basis of a label appended to the packet header. Prior to forwarding of each packet, a path known as label switched path (LSP) is established on the basis of a forward equivalence class (FEC). The FEC is set up depending on several QoS parameters. To set up an LSP two protocols are widely used among the LSRs - an interior routing protocol such as Open Shortest Path First (OSPF) is used to exchange routing information and Label Distribution Protocol (LDP) or Resource Reservation Protocol (RSVP) with traffic engineering extension is used to distribute the labels. Whenever an IP packet enters an MPLS domain, the ingress edge router appends the label in the packet header according to the FEC the LSP matches and sends the packet to the next LSR. The LSR, on receipt of the packet, strips off the incoming label, appends the outgoing label and sends the packet to the next LSR along the LSP. When the packet reaches the egress edge router, it strips off the MPLS header and sends the packet to the destination as specified by the IP address. Thus a significant reduction in switching time 
takes place as the intermediate routers in the MPLS domain forward the packets only on the basis of the label appended to the header.

There are several challenges in applying MPLS to optical networks [12]. The most important of these is the selection of labels. In optical networks a label can be thought of as a wavelength, a waveband or an entire fiber. With the advent of WDM technology, it is preferable to take a wavelength as a label. This is because several lightpaths are multiplexed on a single fiber and a label should uniquely identify a specific lightpath channel on a fiber. The second most important challenge of extending MPLS to optical networks is the fact that zero bandwidth paths cannot be established for future use in an optical network. In an IP network, bandwidth is never used up in a particular path unless data is sent along that path. But this is not the case in an optical network, where a connection is set up by crossconnecting channels using optical cross-connects (OXCs).

As we have discussed in chapter 1, optical network survivability can be either pathbased or link-based. In path-based protection it is the responsibility of the source node to restore the network. Any node detecting a fault, be it either link or node, immediately sends an error message to the source node. This message travels upstream to the source node, which can be very slow due to several reasons. The first is that the error message is intercepted and processed at each node which delays the message to reach the source. Second is that in a WDM network, if a link fails, several lightpaths fail simultaneously. Hence there are several error messages generated at the same time destined to different source nodes, which increase network congestion and take up network resources. In G(Generalized)MPLS, a new message has been included which sends the error message directly to the source node without the intervention of the intermediate nodes. 
This can be avoided if link-based protection is used instead of path-based protection. Here, the node that detects the fault is responsible for restoring the network. Each link has a dedicated or shared back-up path which can be used in case of the primary link failure. However, in spite of the fact that link-based protection is faster than the pathbased one, it suffers from one big disadvantage. It cannot be used in case of a node failure. In our design we have used path-based protection.

There can be another way to restore a failed network. This is by means of a centralized management technique, where a separate management entity is to be present to take over the restoration functionalities. Whenever a node or a link fails, the failure is reported to the centralized manager who computes a backup path and restores the network. However, this approach does not suit a large network as we have already discussed several disadvantages of a centralized network management.

In extending the concept of MPLS to optical network restoration, we should first take a look at the survivability features of MPLS. First, there is MPLS restoration. Restoration typically refers to switching the connection to an alternative path after a link or a component fails in the system. The path may be computed at the time of failure or at the same time as that of the primary path. One of the major disadvantages of MPLS restoration is that it takes into account all the IP switching problems, such as long convergence times, temporary instability, etc. [10]. This problem is proposed to have been overcome by Didier Colle, et. al. [10]. They proposed a restoration scheme known as fast topology-driven constraint-based rerouting [10] which is based upon the assumption that the routing protocol used in MPLS is link-state. Every router shares the current state of each link in the network. When a node or a link fails, the router detecting 
the failure knows that it has to compute an alternate route. It does so after eliminating the failed node or link from its link state table.

The second feature of MPLS survivability is MPLS protection. Protection refers to the preestablishment of an alternate path which is assumed by the connection after a failure in the network. Protection can be typically of two types - local protection and path protection [10]. Local protection refers to preestablishment of a back-up path which spans a single link or a node. Here the node detecting a failure can initiate the survivability mechanism by switching to the alternate path. Path protection refers to establishment of a back-up path which is assumed by the connection when a link or a node fails. The node detecting the failure, sends the information all the way to the source node which initiates the survivability mechanism. Thus, path protection needs an additional switching functionality and takes a longer time to restore a fault than local protection.

While discussing survivability mechanisms in optical networks, it can be noted that there are three major steps to restore a failed lightpath. The first is to select the restoration path itself. This can be compared to any ordinary route selection in an IP network and is a network layer functionality. The second step is to assign wavelengths along that restoration path. The final step is to configure the cross-connects so that a lightpath is established along that restoration route. Each of these functionalities can be done before or after the failure of a lightpath channel [12]. We defined three categories of restoration in our thesis depending upon these functionalities. In category 1 restoration the source node will transmit the service lightpath and the restoration lightpath at the same time. In category 2 , the restoration path will be calculated at the same time as the service path and 
wavelengths will be reserved along that path. After a failure occurs, only the optical cross-connects will be configured to switch the failed connection along the restoration path. The third category of restoration will compute the restoration path at the same time as the service path, but wavelengths will be assigned to that path after failure occurs. When a lightpath fails, the source node will find out the available wavelengths along the pre-computed restoration path and assigns those wavelengths to the restoration lightpath. If no wavelengths are available, a new restoration path is computed and wavelengths are assigned.

These three categories have their relative advantages and disadvantages. Category1 is the fastest method of restoration, but it has the disadvantage that it takes up a lot of network resources. Category 2 also takes up equal amount of network resources. Category 3 is the slowest among the three but it utilizes the available network resources very efficiently. We will try to optimize between these different categories in order to utilize the available network resources efficiently as well as to make the restoration method fast.

\subsection{Design}

\subsubsection{Concept and assumptions}

In our thesis we developed a method to restore a failed lightpath which, on one hand, provides a fast restoration method to suit real-time applications and, on the other hand, utilizes the network capacity efficiently, thus reducing the cost of network restoration. It is expected that the viable solution to this problem will be an optimization between cost and speed which will make a WDM optical network suitable for real-time 
multimedia traffic. While designing the proposed fault restoration algorithm, there are two major problems that need to be addressed. The first is the high network restoration cost due to large wavelength requirement in case of predesigned protection. This is required because a separate restoration path has to be set up for each service lightpath, which takes up a large number of wavelengths and increases cost in the case of predesigned protection. The second is the large network restoration time required in case of dynamic restoration when a restoration path is set up and wavelengths assigned after the occurrence of a failure. Our method tried to optimize between these two in order to reach a viable solution to this problem.

We have three major assumptions in our design. The first is that all the nodes in the network have wavelength conversion capability. That is, all the nodes can convert any input wavelength to any output wavelength. The second assumption is that all faults in the network are either single link fault or single node fault. The third assumption is that all the links used in the network are bi-directional.

In the context of these assumptions, it may be noted that the basic failures in a WDM optical network are of three types [1]. These are link failure, node failure and channel failure. While the link and node failure are concerned with the failures of a link or a node, the channel failure is concerned with the failure of a transmitting or receiving equipment operating at that particular channel. In our design, we are primarily concerned with a single link or a single node failure. This gives us a simplistic planning for the failure recovery. Also, since a cable cut is the most common type of failure in an optical network, this assumption gives us a strong foundation for our design. 
Our design tried to extend the concept of forward equivalence class (FEC) of MPLS to restore a failed lightpath. As discussed earlier, the basic functionality of MPLS operation is based upon the fact that traffic in MPLS is grouped into FECs. Grouping of traffic into FECs depends upon several factors some of which are: destination address, source address, source and destination ports, IP protocol ID and IPV6 flow label [12]. When an ingress edge router in an MPLS domain gets a packet, it first determines the FEC to which the packet belongs, according to which it switches the packet along a particular LSP. In our design, each node maintains a table called FEC table where paths and corresponding wavelengths to all the destinations are stored according to connection types. Each connection type corresponds to a particular FEC. Table 3.1 shows a sample of the FEC table at node 1.

\begin{tabular}{|c|c|c|c|}
\hline Destination node & Connection type & Path & Wavelength \\
\hline 6 & 1 & $1-2-3-4-5$ & w4-w6-w4-w4-w4 \\
6 & 2 & $10-11-12-13-14$ & w5-w5-w5-w5-w5 \\
6 & 3 & $19-20-21-22-23$ & w5-w5-w5-w5-w5 \\
\hline
\end{tabular}

Table 3.1. Sample of FEC Table at node 1

Whenever a node gets a lightpath request, it sets up the service lightpath for that request according to the FEC the lightpath matches. Paths and wavelengths are assigned to that service path corresponding to the FEC the path belongs to. The node then goes to 
set up the restoration path for that service path according to the algorithm that we proposed which will be discussed in the subsequent sections.

In addition to the FEC table, each node maintains two other tables. The first of these is the status information table. While designing our restoration algorithm, we assumed that network management functionalities would be distributed, instead of being centralized. This has several advantages. The huge load on the central database server can be avoided by distributing the information to all the nodes. The central dependability can also be avoided by introducing multiple failure points. We assume in our design that all the nodes share the same status information which is distributed among them using any well known message distribution protocol. Thus each node knows all the information about all other lightpaths originating and terminating at any node pair as well as of their restoration paths. Table 3.2 shows a sample of the status information at each node.

\begin{tabular}{|c|c|c|c|l|l|l|l|}
\hline $\begin{array}{l}\text { Light- } \\
\text { path }\end{array}$ & $\begin{array}{l}\text { Source } \\
\text { node }\end{array}$ & $\begin{array}{l}\text { Destination } \\
\text { node }\end{array}$ & $\begin{array}{l}\text { Links } \\
\text { used }\end{array}$ & $\begin{array}{l}\text { Wave- } \\
\text { lengths } \\
\text { used }\end{array}$ & $\begin{array}{l}\text { Restoration } \\
\text { path }\end{array}$ & $\begin{array}{l}\text { Restoration } \\
\text { links }\end{array}$ & $\begin{array}{l}\text { Restoration } \\
\text { wave- } \\
\text { lengths }\end{array}$ \\
\hline LP1 & 1 & 6 & $\begin{array}{l}1-2- \\
3-4-5\end{array}$ & $\begin{array}{l}\text { w4- } \\
\text { w6- } \\
\text { w4- } \\
\text { w4-w4 }\end{array}$ & RP1 & $\begin{array}{l}\text { 19-20-21- } \\
22-23\end{array}$ & $\begin{array}{l}\text { w12-w12- } \\
\text { w6-w6-w2 }\end{array}$ \\
\hline
\end{tabular}

Table 3.2. Sample of status information at each node

Finally, a node should also have prior knowledge to all the free wavelengths available on each link. For this, we introduce another table called the Free Wavelength table. Whenever a source node allocates wavelengths to a service path as well as to a 
restoration path, it updates its free wavelength table and distributes this information to all other nodes which subsequently update their free wavelength tables. Table 3.3 shows a sample of a free wavelength table available at each node.

\begin{tabular}{|c|c|}
\hline Links & Free wavelengths \\
\hline 1 & w1 w2 w3 w5 w6 w7 w8 w9 w10 w11 w12 w13 w14 w15 \\
2 & w1 w2 w3 w5 w6 w7 w8 w9 w10 w11 w12 w13 w14 w15 \\
\hline
\end{tabular}

Table 3.3. Free wavelength table at each node

As we have discussed earlier, a WDM mesh network can be protected in two ways. First is predesigned protection, where, a backup lightpath is computed and wavelengths reserved at the same time of the service path. The second is dynamic restoration, where, a backup path is computed at the same time of the service path but wavelengths are assigned after failure occurs. The first method is very fast, but it takes up a lot of network resources. The second method is comparatively slower but utilizes network resources very efficiently. To design a fault-tolerant WDM network suitable for a real-time application, it is important to make the fault restoration method very fast. But if we design the network in such a way that all the service lightpaths are protected by a restoration path with wavelengths reserved, then the network cost will be very high as it will require a large number of wavelengths to protect that network. On the other hand, if we design the network such that it is protected by dynamic restoration method, the network would utilize the available resources efficiently, no doubt, but it would not suit 
real-time multimedia traffic as the restoration time will be higher. To optimize between the network restoration time and cost, we have introduced a priority field in the packet header. The priority field will enable the source node to decide which restoration method to use - predesigned protection or dynamic restoration. We have categorized the priority field into three types - high, medium and low. If a connection request is of high priority, then the source node uses category 1 restoration. It computes the backup lightpath at the same time of the service lightpath, assigns wavelengths to it and transmits the signal both on the service and backup lightpaths at the same time. On the other hand, if a connection request is of medium priority, the source node uses category 2 restoration, where, it computes the backup lightpath at the same time as that of the service lightpath and reserves wavelengths along that path. On the occurrence of a failure, the source node simply switches to the backup path. For the lowest priority connection request, the source node uses category 3 restoration, where, it computes the backup path at the same time of the service path, but assigns wavelengths to it after a failure occurs. In the next section we would discuss our algorithms for the above restoration methods.

\subsubsection{Algorithms}

We have initially designed our algorithms based on a WDM mesh network with 14 nodes, 23 bi-directional links and 15 wavelengths per link. Fig. 3.1 shows the network. 


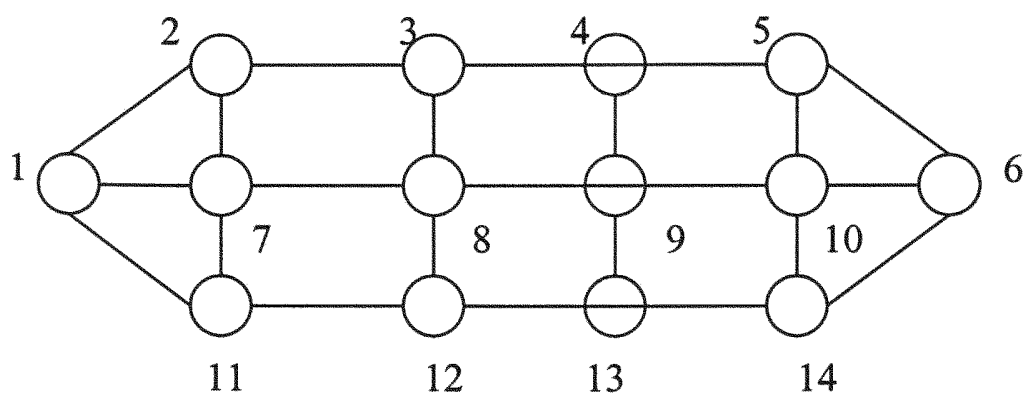

Fig. 3.1 WDM mesh network used in the design

The nodes are numbered from 1 to 14 , links are numbered from 1 to 23 and wavelengths are numbered from w1 to w15.

Our algorithm starts when a node gets a lightpath request. It first sets up the service path according to the FEC which the lightpath matches. It then determines the priority of the connection according to the priority field. The restoration method is determined accordingly. Our algorithm for categoryl restoration works as follows: 


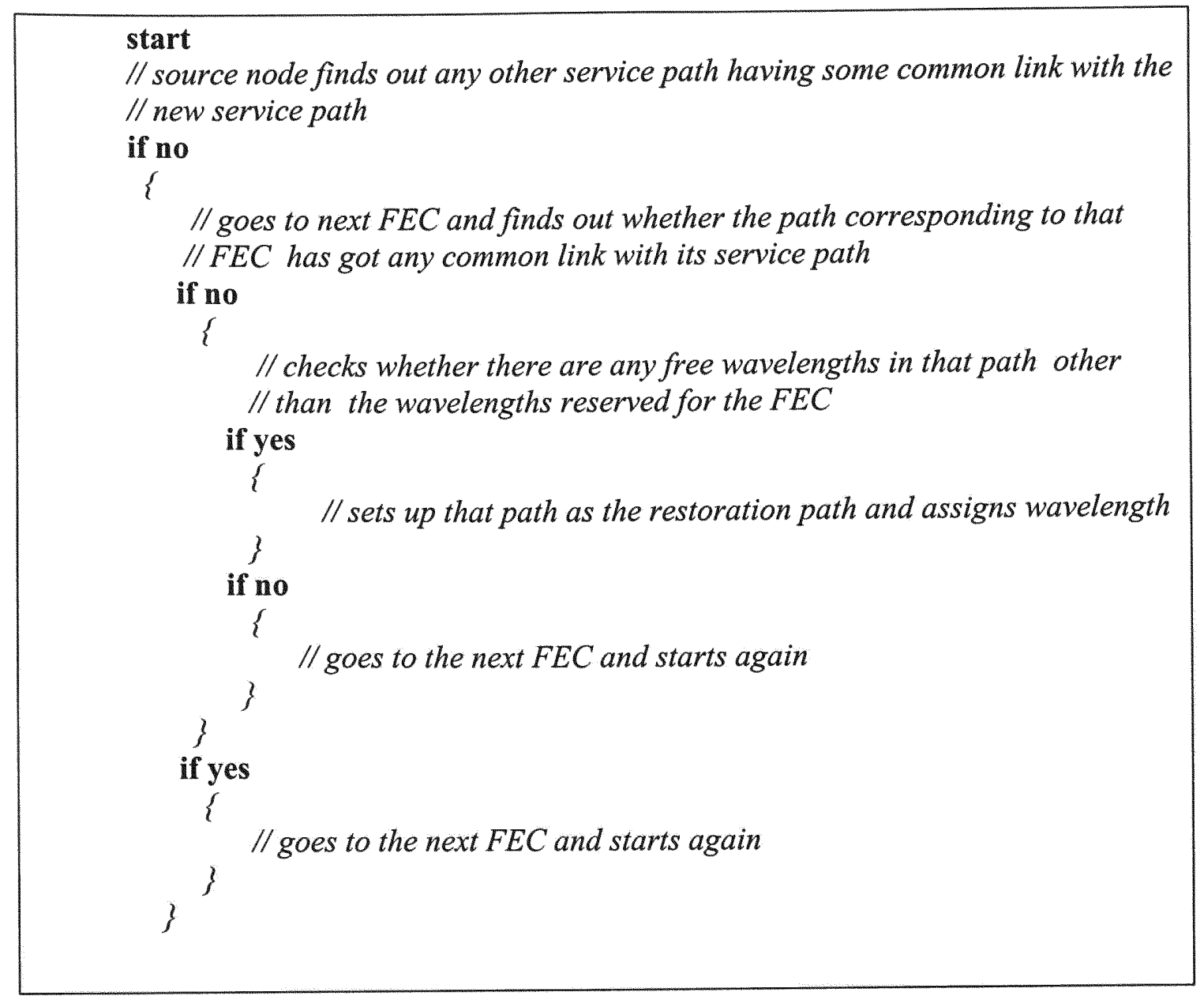

The above algorithm works when the source node finds that no other service path has any common link with the new service path. If it finds that the new service path has one or more common links with any existing path, it finds out the restoration path as follows: 


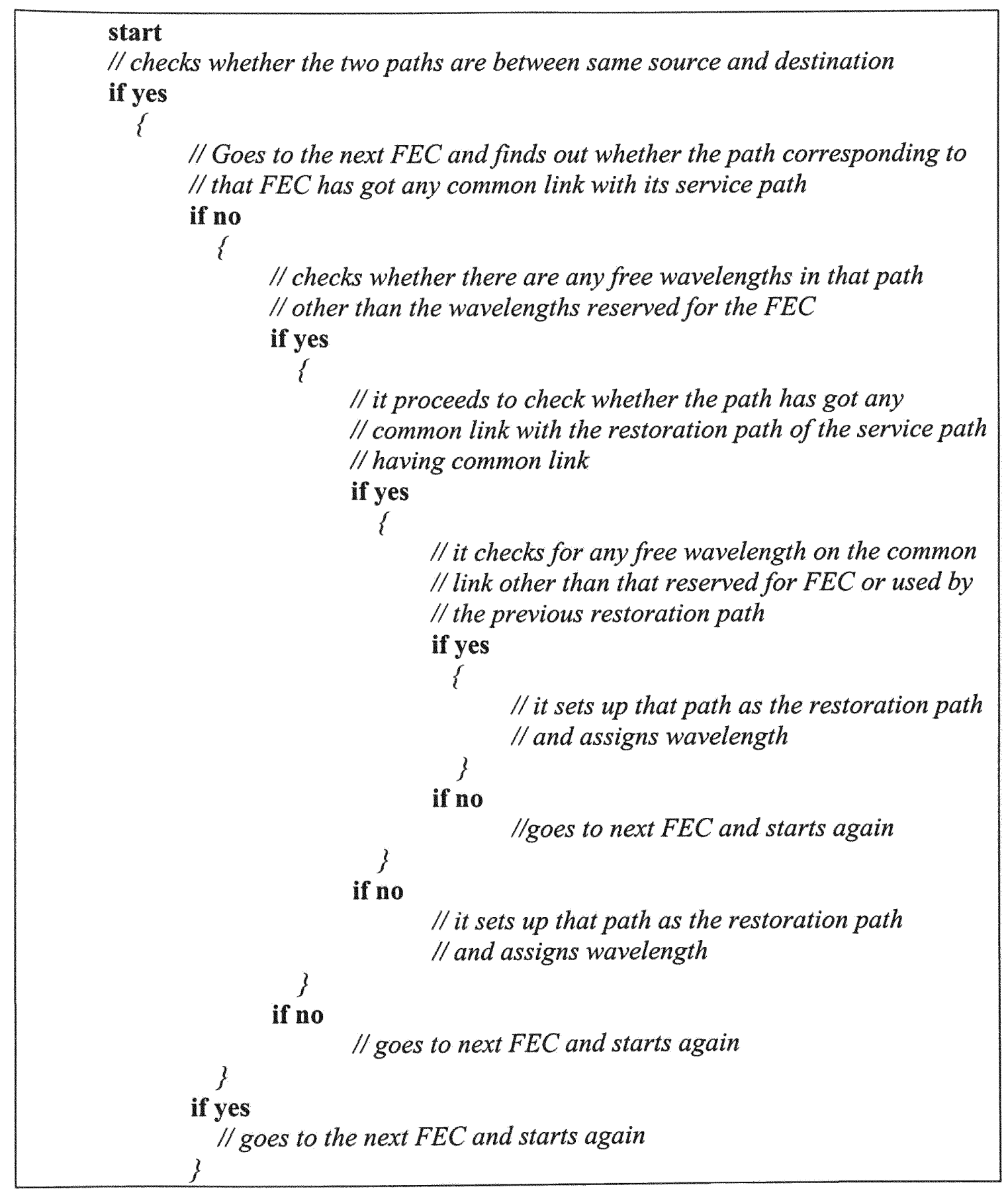

The above algorithm works if the source node finds that the two service paths are between same source and destination. If they are not between same source and 
destination, the source node finds out the restoration path corresponding to the service path and proceeds to set up the new restoration path exactly as described above.

The algorithm we just now described work for categoryl restoration. The same algorithm will also work for category2 restoration. However, the method to restore a network for category 3 restoration is completely different. In this case, upon occurrence of a failure, the source node assigns free wavelengths to the pre-computed restoration path using any wavelength assignment algorithm. If there is not enough free wavelength to assign, it computes another restoration path and assigns wavelengths. Thus the problem here boils down to the well-known routing and wavelength assignment (RWA) problem.

The algorithm discussed above works as follows: a source node, on receipt of a connection request and after finding out that category1/category 2 restoration to be used, finds out from its status information table whether there is any other service path having some common link with the new service path. If there is none, it searches its FEC table to find a path which is link-disjoint from the new service path. It then consults the free wavelength table to find any free wavelength on the link-disjoint path other than that reserved for the FEC. It sets up that path as the restoration path and assigns the wavelengths. When a lightpath request comes later that matches that $\mathrm{FEC}$, the source node selects the first service path (which is link-disjoint) as the restoration path and assigns wavelengths from the free wavelength table.

If the source node finds that there is another service path having one or more common link with the new service path, it performs two rounds of check. First, it checks the FEC table to find a link-disjoint path, assigns wavelengths to it from the free wavelength table. Then, it goes on to check whether the path has any common link with 
the restoration path of the service path having common link. If not, it sets that path as the restoration path and assigns wavelengths. If there is one or more common links, it has to assign wavelengths other than that used up by the previous restoration path.

After computing a service path and restoration path, the source node updates its status info and free wavelength tables. It then shares the information with all other nodes so that they can update their respective tables also. Thus, as soon as each node gets a lightpath request and updates its tables after computing the paths and assigning wavelengths, it sends out control messages to all other nodes in order to enable them to update their respective tables.

FEC of a lightpath is determined keeping in mind several factors. Some of them are destination IP address, destination port, etc. All the lightpath connections are categorized into different FECs and the information is distributed to all the nodes. The nodes subsequently set up their respective FEC table from the distributed information. 


\section{Chapter 4 Simulation and Results}

\subsection{Simulation scenario}

Our simulation is based on a WDM mesh network having 14 nodes, 23 bidirectional links and 15 wavelengths per link. The network is shown below.

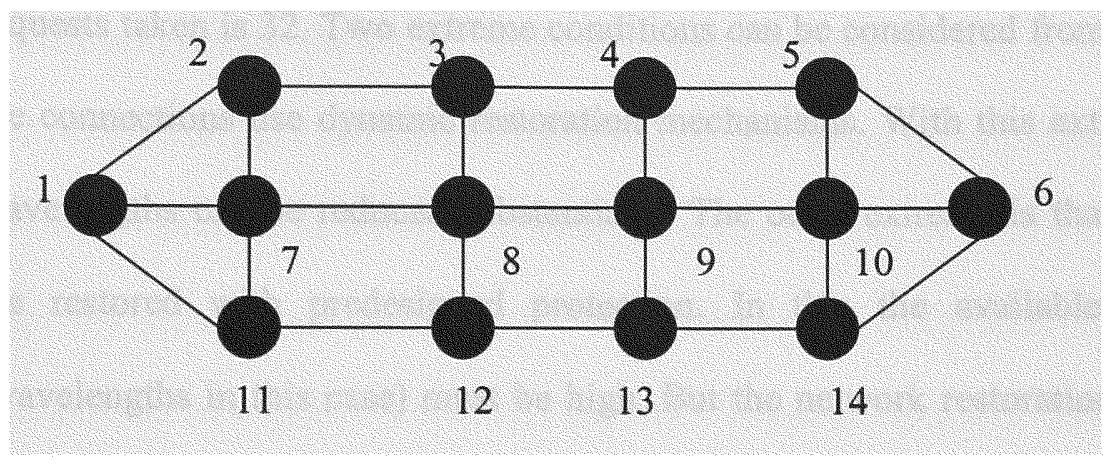

Fig. 4.1 WDM mesh network

The network traffic is assumed to be dynamic. Whenever a node boots up in the network, its FEC table is configured. This FEC table has been assumed to be a precomputed and static one. This is in line with the concept of FEC in MPLS where each connection is switched according to labels predetermined by a particular FEC. The route for each type of lightpath has been determined in priori and wavelengths are also reserved. Whenever a lightpath request matches a particular connection type, it is assigned a route along with the wavelengths determined from the FEC table.

While running the proposed algorithm, we found out that the minimum number of wavelengths that could be taken in each link is twelve. If the number of wavelengths is 
taken to be less than twelve per link, then it becomes difficult to configure the FEC table. The FEC table that we designed has a variable number of different connection types to each node in the network. The minimum number of connection types is taken to be 2 and the maximum is 4 . Theses are experimental numbers and are taken for the sake of simulation simplicity.

While running the algorithm at node 1 , the maximum number of static connection requests taken is 32 . Two extreme conditions can be considered from here. One is that all the connections use dynamic restoration mechanisms. With this extreme, the number of wavelengths can be reduced substantially. The other extreme is that all the connections are restored with predesigned protection. In this the available network resources (wavelengths in this case) must be high. But the network restoration can be very fast as compared to dynamic restoration. Hence we propose an optimization between restoration cost and speed by introducing a priority field in the packet header.

As we have already discussed, there are three main assumptions in our design. The first is that all the nodes in the network have wavelength conversion capabilities. Wavelength converters are costly, but they are readily available nowadays and can be easily configured in present day networks. Having wavelength conversion capabilities in all the nodes increases the cost of the network but substantially reduces the blocking probability at each node. As any incoming wavelength can be converted to any outgoing wavelength, the speed of restoration also increases. The second assumption is that all the faults in the network are either single link fault or single node fault. Thus, while running the algorithm, we assumed that only one node or link fault can occur at any point of time. Thus, when one failure takes place, the failure that preceded immediately has already 
been restored. The third assumption is that all the links used in the network are bidirectional. This significantly reduces the number of links in the network, thus reducing the computational complexity.

\subsection{Results}

We used Microsoft Visual $\mathrm{C}++$ for our simulation. The algorithm starts with initialization of all the tables. When a lightpath request arrives, it sets up the service path and restoration path and assigns wavelengths. We tested the algorithms in computers with Pentium 4 processors having $2.2 \mathrm{GHz}$ processing speed. We have divided the algorithms into two parts - one for finding a restoration path when there are no common links between the new primary path and any other existing lightpath; the second is for finding the restoration path if some common links are found. We tested the algorithm by first giving a lightpath request between nodes 1 and 6 . We found a restoration path set up time of 1.25 microseconds on the above processor. After the first connection was set up, the status info table and the free wavelength table were configured, and another lightpath request between nodes 1 and 5 was given. The time was found to be 1.4 microseconds. Another test was carried out by giving a lightpath connection between nodes 1 and 6 . Time was found to be 1.5 microseconds. Thus, an average restoration time of 1.38 microseconds was found. These three cases were tested for the first type of algorithm where there are no common links between a new lightpath and existing lightpaths. After configuring the status info and free wavelength tables, another new lightpath request is given between nodes 1 and 6 . This is for the type 2 algorithm where there are common 
links between the new lightpath and existing lightpaths. It was found that the restoration path set up time is a little higher, in the order of 14 microseconds.

The algorithms are once again tested in a computer with Pentium 4 processor with $1.6 \mathrm{GHz}$ processing speed. Similar connection requests were given. For the type 1 algorithm, successive times were found to be 1.87 microseconds, 1.88 microseconds and 2.03 microseconds with an average time for restoration path set up of 1.9 microseconds. However for type 2 algorithm restoration path set up time is 21 microseconds.

Same set of tests are carried out on a Pentium 3 machine with processing speed of $900 \mathrm{MHz}$. Average restoration path set up time for type 1 algorithm was found to be 2.11 microseconds. For type 2 algorithm where there are common links between a new lightpath and existing lightpaths, restoration path set up time was found to be of the order of 30 microseconds. The graph below summarizes the results.

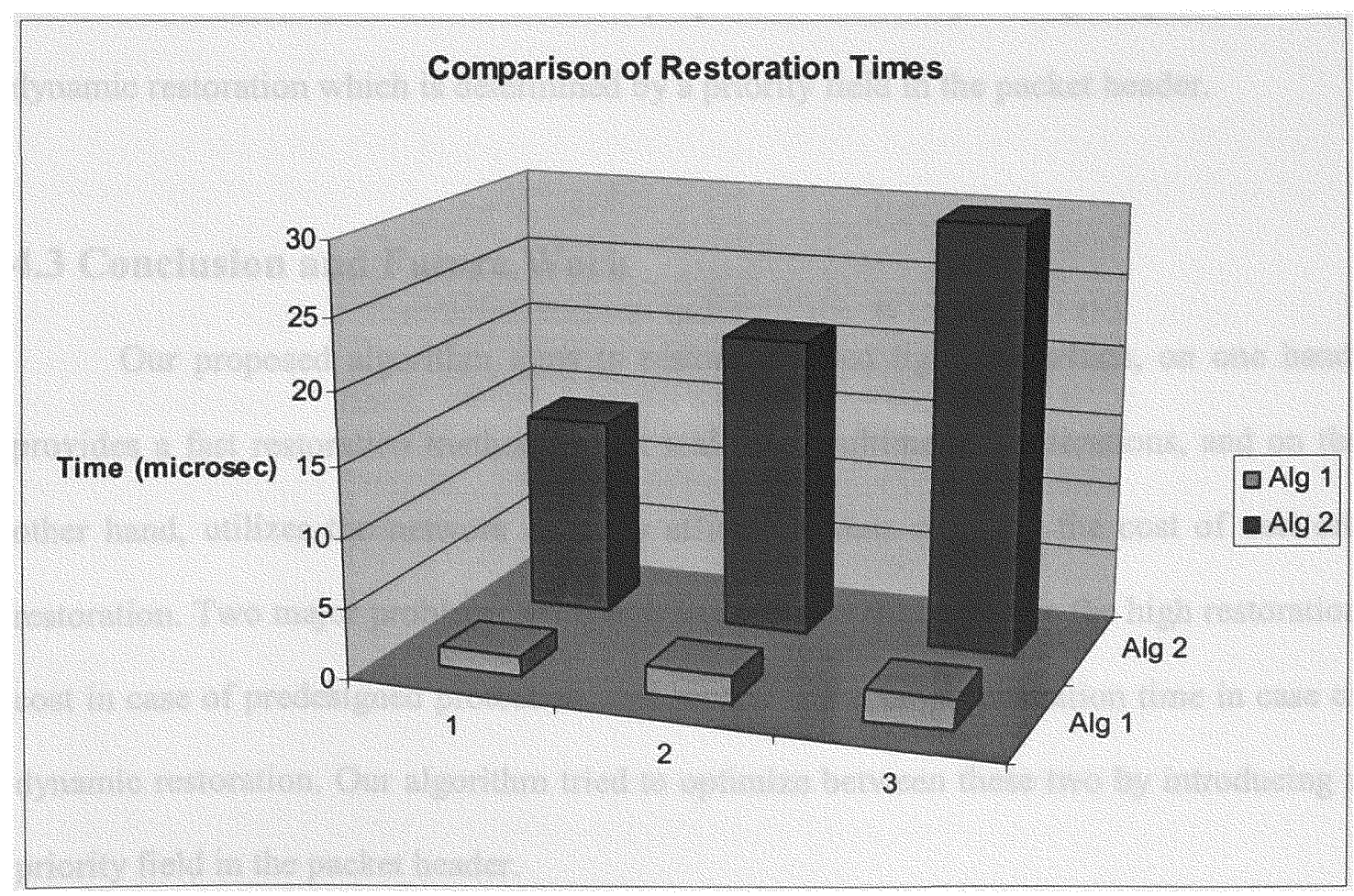


Although our simulation is based on a small network with 14 nodes and 23 bidirectional links, it can very well be extended to any real-life network with thousands of nodes. Although we have not tested the algorithm in a real-life network, yet we can very well predict that it will give the desired results.

We have set up 32 different connection types from node 1 to all other nodes which are configured in the FEC tables. Thus it can be thought of as a static network with known lightpath requests. If we want to protect all theses lightpath requests with predesigned protection, then we would have needed a lot more wavelengths per link which would make the network very costly. In that case, our choice of 15 wavelengths per link would result in the protection of a very few lightpaths, making most of them nonrecoverable. On the other hand, if all the lightpath connections are to be protected with dynamic restoration, then the increased time of protection makes the network unsuitable for real-time multimedia traffic. Thus we propose to use both predesigned protection and dynamic restoration which is determined by a priority field in the packet header.

\subsection{Conclusion and Future Work}

Our proposed algorithm aims to restore a failed lightpath which, on one hand, provides a fast restoration method to suit real-time multimedia applications, and on the other hand, utilizes the network capacity efficiently thus reducing the cost of network restoration. Two major problems were addressed in this study: first is the high restoration cost in case of predesigned protection, and second is the large restoration time in case of dynamic restoration. Our algorithm tried to optimize between these two by introducing a priority field in the packet header. 
Our simulation is based on a small network with 14 nodes and 23 bi-directional links. Although we have not extended our simulation to any real-life network with thousands of nodes, we are sure that it would also give satisfactory results when extended.

Our design is based on FEC tables which are pre-configured at each node. Thus we have considered only static traffic demand. This can be extended to dynamic traffic demand also where some amount of intelligence is extended in each node to deal with dynamic changes in traffic. This can be an interesting future traffic engineering extension.

Our work mainly deals with predesigned protection. Much research needs to be done on dynamic restoration which can be a future extension of this research. 


\section{Bibliography}

[1] Dongyun Zhou and Suresh Subramaniam, "Survivability in Optical Networks", IEEE Network, Nov/Dec 2000

[2] Maode Ma and Mounir Hamdi, "Providing Deterministic Quality of Service Guaranties on WDM Optical Networks", IEEE Journal on Selected areas in Communication, vol.18, no.10, October 2000

[3] Olivier Crochat, Jean-Yves Le Boudec and Ornan Gerstel, "Protection Interoperability for WDM Optical Networks", IEEE/ACM Transaction on Networking, vol. 8 , no. 3, June 2000

[4] Kevin H. Liu, Brian J. Wilson and John Y. Wei, "A Management and Visualization Framework for Reconfigurable WDM Optical Networks”, IEEE Network, Nov/Dec 2000

[5] Gurusamy Mohan and C Siva Ram Murthy, "Lightpath Restoration in WDM Optical Networks", IEEE Network, Nov/Dec 2000

[6] Andrea Fumagalli and Luca Valcarenghi, "IP Restoration Vs WDM Protection:Is There an Optimal Choice?", IEEE Network, Nov/Dec 2000

[7] Sophie De Maesschalck, Didier Colle, Adelbert Groebbens, Chris Develder, Ilse Lievens, Paul Lagasse Mario Pickavet, Piet Demeester, Fausto Saluta and Marco Quagliotti, "Intelligent Optical Networking for Multilayer Survivability", IEEE Communications Magazine, Jan 2002

[8] S. RamaMurthy and Biswanath Mukherjee, "Fault Tolerant Design of Wavelength Routed Optical Networks", DIMACS series in Discrete Mathematics and Theoretical Computer Science, vol. 48, pp. 117-128, March 1998

[9] Laxman Sahasrabuddhe, S Ramamurthy and Biswanath Mukherjee, "Fault Management in IP over WDM Networks:WDM Protection Vs IP Restoration", IEEE Journal in Selected Areas in Communications, vol. 20, no. 1, January 2002

[10] Didier Colle, et al., "Data Centric Optical Networks and Their Survivability", IEEE Journal in Selected Areas in Communications, vol. 20, no. 1, January 2002

[11] Guangzhi Li, Jennifer Yates, Dongmei Wang and Charles Kalmanek, "Control Plane Design for Reliable Optical Networks", IEEE Communications Magazine, February 2002

[12] Robert Doverspike and Jennifer Yates, "Challenges for MPLS in Optical Network Restoration", IEEE Communications Magazine, February 2001 
[13] Makki K., Pissinou N. and Gumaste A., "A Hybrid Method to Cellular Traffic Using Optic Beam Interconnects", in Proceedings of IEEE Wireless Communications and Networking Conference, Chicago, Illinois, September 23-28, 2000

[14] William Stallings, "MPLS”, IP Journal, vol. 4, no. 3, September 2001

[15] Rajiv Ramaswami and Kumar N. Sivarajan, "Optical Networks A Practical Perspective", Morgan Kaufmann Publishers , Inc., 1998 